\title{
An Efficient and Simple Switching Filter for Removal of High Density Salt-and-Pepper Noise
}

\author{
Hani M. Ibrahem \\ Math and Computer Science dept., Faculty of science, Menufyia university, Egypt. \\ hanimir78@yahoo.com
}

\begin{abstract}
This paper presents an efficient and simple adaptive method for high density salt-and-pepper noise removal. A noise detector is utilized to check whether the selected pixel is noisy or noise free. Noise pixels will then be subjected to the second stage of the filtering action, while the noise free pixels are left unaltered. Since not every pixel is filtered, undue distortion can be avoided. The noise free pixels are only considered in the filter operation for finding the value of the processed pixel. The window size is selected as 3 X 3 in the first step. If all pixels within the window are considered to be noise, then change the selected window size to $5 \mathrm{X}$ 5. If all the pixels within the selected $5 \times 5$ window are considered to be noise, then the processing pixel is replaced by the previous resultant pixel. This technique requires one nonnoise original image as training image. The key point of the filter operation is based on the solution of the equations system $X=A^{-1} B$ in the nonnoise original image. An algorithm to extract the data from the nonnoisy image and form it in the linear equation system is presented. Comparison of the given filter with other existing filters is provided in this paper. The results demonstrate that the proposed technique can obtain better performances than other existing denoising techniques. The proposed method works well for high-density salt $\&$ pepper noise even up to a noise density of $97 \%$.
\end{abstract}

Index Terms - Salt - and - pepper noise, switching filter, noise reduction, image denoising.

\section{INTRODUCTION}

Digital images are corrupted by undesirable random variations in intensity values called noise [1]. During image acquisition or transmission, digital images could be contaminated by impulse noise, which is caused by malfunctioning pixels in camera sensors, faulty memory locations in hardware, or transmission in a noisy channel [2]. It's important to eliminate noise before subsequent image processing tasks such as edge detection or segmentation is carried out. Salt-andpepper noise is a special case of impulse noise where the noisy pixels can take only the maximum and minimum values in the dynamic range. Recently, many algorithms such as [2-6] have been proposed to carry out the salt-and-pepper noise suppression. Therefore, to efficiently remove noise from an image while preserving its features is a fundamental problem of image processing [7]. The standard median filter (SMF) is the most popular choice for removing the salt-andpepper noise. However, the median filter and its derivations such as weighted median (WM) [8], center weighted median (CWM) [9], and recursive weighted median (RWM)[10] are typically implemented invariantly across an image. They tend to alter the noise free pixels as well as the noise pixels, exhibits blurring of filtered images. To avoid the damage on the noise free pixels, many image filters with an impulse detector are proposed such as in the literatures [4],[1113].

In this paper, an efficient and simple method to remove salt-and-pepper noise is proposed. In this method, only the noise pixels are changed by filter operation, while the noise free pixels are kept unchanged. In addition, the window size is altered among 3 X3 and 5X5 based on the noise and noise free pixels within the window. This technique requires only one original nonnoise image as data set. May be the median value or other values based on the median are not the correct choice for noise pixel replacement. Thus, the filter output is based on the solution of the system $\mathrm{X}=\mathrm{A}-1 \mathrm{~B}$ in the nonnoise original image which will be explained later. Further, this method does not require threshold parameters. This method can remove saltand-pepper-noise with a noise level as high as 97\%, and it's relatively fast. The experimental results demonstrate that the proposed method is effective for removing noise and preserving fine details than other existing methods. The outline of this paper is organized as follows. A brief review of related work is given in section II. In section III, the proposed technique is introduced. The implementation results and comparison are provided in section IV. Finally, the conclusions are summed up in section V.

\section{RELA TED WORK}

Many approaches have been proposed to removesalt and-pepper noise. Chang-you et al [13] have proposed an adaptive weighted median filtering algorithm to remove salt -and- pepper noise. This algorithm first takes a decision whether the pixel under test is noise or not by comparing the block uniformity of the $3 \times 3$ 
window with one of the entire image, then adjusts the size of filtering window adaptively according to the number of noise points in the window. Further, Toh and Isa [11] have proposed two-stage noise adaptive fuzzy switching median (NAFSM) filter for salt-and-pepper noise detection and removal. The detection stage will use the histogram of the corrupted image to identify noise pixels. These detected the noise pixels will and then by filtering action, while noise free pixels are retained and left unprocessed. Then, the NAFSM filtering mechanism employs fuzzy reasoning to handle uncertainty present in the extracted local information as introduced by noise. Haidi et al. [14] have proposed a technique to remove impulse noise from highly corrupted images. The method is actually a hybrid of the adaptive median filter with the switching median filter. The method adaptively changes the size of the median filter based on the approximation of local noise density. Haidi et al [14] claimed that their method is better than the works in [15-17]. In addition, K. S. Srinivasan and D. Ebenezer [18] have proposed a decision-based algorithm (DBA) for restoration of images that are highly corrupted by impulse noise. This algorithm shows significantly better image quality than a standard median filter (SMF), adaptive median filters (AMF), a threshold decomposition filter (TDF), cascade, and recursive nonlinear filters. This method processes the corrupted image by first detecting the impulse noise. The detection of noisy and noise-free pixels is decided by checking whether the value of a processed pixel element lies between the maximum and minimum values that occur inside the selected window. This is because the impulse noise pixels can take the maximum and minimum values in the dynamic range $(0,255)$. If the value of the pixel processed is within the range, then it is an uncorrupted pixel and left unchanged. If the value does not lie within this range, then it is a noisy pixel and is replaced by the median value of the window or by its neighborhood values. Furthermore, Cangju Xing [5] has proposed a method for removing heavy salt-and pepper noise. This method includes three steps. In the first step, the noise pixels are distinguished from the signal pixels; then set initial values for noise pixels; finally, compute the output. The main difference from other switch-type filters is the means to change the values of the contaminated noise pixels. Furthermore, a modified decision based unsymmetrical trimmed median filter algorithm (MDBUTMF) for highly corrupted image by salt -and- pepper noise is proposed in [19]. This algorithm replaces the noisy pixel by trimmed median value when other pixel values, 0 's and 255's are present in the selected window and when all the pixel values are 0 's and 255's then the noise pixel is replaced by mean value of all the elements present in the selected window.

\section{PROPOSED METHOD}

An efficient method for removal high density salt and - pepper noise with preserving image details is proposed. It is composed of three stages as: (1) Data extraction from the nonnoisy image. (2) Noise detection. (3) Noise cancellation. Figure.1 shows the core idea for the proposed method. The extracted data from the nonnoisy image, as Lena image, is applied in the filter equation to produce filtered image from its noisy image.

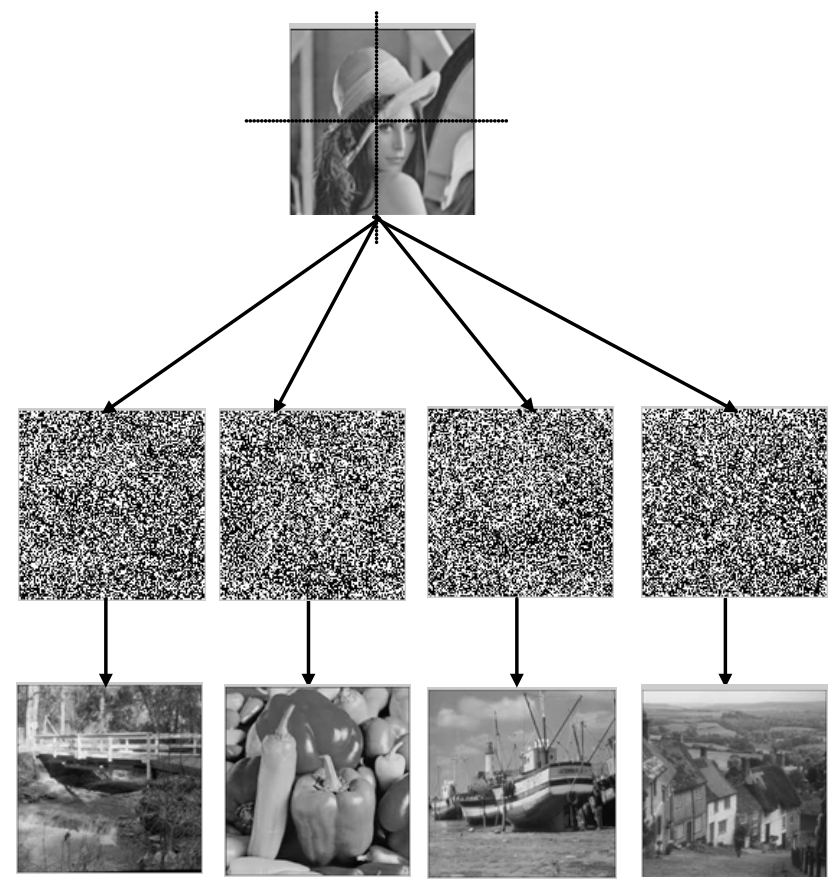

Figure.1. The Core Idea for the Proposed Method.

\section{(1) Data extraction}

Extracted the data from the nonnoisy image are used in the filter operation to produce filtered image from its noisy image. This is presented in this stage by achieving the following steps:-

1- Divide the nonnoisy image into non overlapping four blocks.

2- $\quad$ Repeat the following for each block $i \quad \ni i=\overline{1,4}$

2.1-Select 3X3 window and place it, randomly, in any area within the block i.

2.2-Extract the center pixel value and put it in the first row in the vector $B_{i}$.

2.3-Extract two nearest neighbors and put them in the first row in matrix $A_{i}$

2.4-Move, randomly, the window within the block $i$.

2.5-Extract the center pixel value and put it in the second row in the vector $B_{i}$

2.6-Extract two nearest neighbors and put them in the second row in the matrix ${ }_{i}$.

End

$$
\text { 3- Compute } A=\left(\sum_{i=1}^{4} A_{i}\right) / 4 \text { and } B=\left(\sum_{i=1}^{4} B_{i}\right) / 4
$$


4- Repeat the following for each block $i$

4.1- Select 3X3 window and place it randomly in any area within the block $i$.

4.2- Extract the center pixel value and put it in the first row in the vector $\bar{B}_{i}$.

4.3-Extract three nearest neighbors and put them in the first row in the matrix $\bar{A}_{i}$

4.4-Move randomly the window within the block $i$.

4.5- Extract the center pixel value and put it in the second row in the vector $\bar{B}_{i}$

4.6-Extract three nearest neighbors and put them in the second row in the matrix $\bar{A}_{i}$.

4.7-Move randomly the window within the block $i$.

4.8- Extract the center pixel value and put it in the third row in the vector $\bar{B}_{i}$

4.9-Extract three nearest neighbors and put them in the third row in the matrix $\bar{A}_{i}$.

End

5- Compute $\bar{A}=\left(\sum_{i=1}^{4} \bar{A}_{i}\right) / 4$ and $\bar{B}=\left(\sum_{i=1}^{4} \bar{B}_{i}\right) / 4$

6- Repeat the following for each block $i$

6.1-Select 3X3 window and place it randomly in any area within the block $i$.

6.2-Extract the center pixel value and put it in the first row in the vector $\hat{B}_{i}$.

6.3-Extract four nearest neighbors and put them in the first row in the matrix $\hat{A}_{i}$

6.4-Move randomly the window within the block $i$.

6.5-Extract the center pixel value and put it in the second row in the vector $\hat{B}_{i}$

6.6-Extract four nearest neighbors and put them in the second row in the matrix $\hat{A}_{i}$.

6.7-Move randomly the window within the block $i$.

6.8-Extract the center pixel value and put it in the third row in the vector $\hat{B}_{i}$

6.9-Extract four nearest neighbors and put them in the third row in the matrix $\hat{A}_{i}$.

6.10-Move randomly the window within the block $i$.

6.11- Extract the center pixel value and put it in the fourth row in the vector $\hat{B}_{i}$
6.12-Extract four nearest neighbors and put them in the fourth row in the matrix $\hat{A}_{i}$.

End

$$
\text { 7- Compute } \hat{A}=\left(\sum_{i=1}^{4} \hat{A}_{i}\right) / 4 \text { and } \hat{B}=\left(\sum_{i=1}^{4} \hat{B}_{i}\right) / 4
$$

All the matrices and vectors that extracted in this stage are used in filter operation.

(2) Noise detection.

Noise detection is the first stage in this method. The salt-and-pepper noise pixels can take the maximum and minimum values in the dynamic range. Therefore, if the value of the processed pixel lies between the maximum and minimum values inside the window, then it kept unchanged. If the value does not lie within this range, then it is a noisy pixel and is replaced by the output of the filter operation.

(3) Noise cancellation

Each element in the vectors $B, \bar{B}$ and $\hat{B}$ is the desired value for its corresponding row (its neighbors) in the matrices $A, \bar{A}$ and $\hat{A}$, respectively. The goal is to estimate parameters which are obtained the desired values, in vectors, from their corresponding rows in the matrices. The linear systems $A X=B, \quad \bar{A} \bar{X}=\bar{B}$ and $\hat{A} \hat{X}=\hat{B}$ are used to describe the relation between the vectors and their corresponding matrices such that $X=A^{-1} B, \quad \bar{X}=\bar{A}^{-1} \bar{B}$ and $\hat{X}=\hat{A}^{-1} \hat{B} . \quad$ The vectors $X, \bar{X}$ and $\hat{X}$ represent the estimation parameters that are used in the filter operation. Switching between $3 \mathrm{X} 3$ and 5X5 window size is used based on the number of noisy pixels where only the noise free pixels are considered in the filter operation. But, if all the pixels inside the 5X5 window are noisy, then replace the processed pixel by the previous resultant pixel. The noise cancellation stage for each pixel location $(i, j)$ to produce filter image $g$ can be achieved by the following steps.

1- Select a 2-D window of size 3X3.

2- Eliminate the noisy pixels within the window.

3- Insert the noise free pixels into vector $R$.

4- Assign the number of pixels in the $R$ to $L$.

5- If $(L<1)$, go to step 10.

6- If $(L=1)$

6.1 Let $g(i, j)$ equal to the pixel value in $L$

6.2 Return to step 1 for the next $(i, j)$

7- If $(L=2)$

$7.1 g(i, j)=L(1) \cdot X(1)+L(2) \cdot X(2)$

7.2 Return to step 1 for the next $(i, j)$

8- If $(L=3)$

$8.1 \quad g(i, j)=L(1) \cdot \bar{X}(1)+L(2) \cdot \bar{X}(2)+L(3) \cdot \bar{X}(3)$ 
8.2 Return to step 1 for the next $(i, j)$

9- If ( $L \geq 4$ )

$$
\begin{array}{rl} 
& g(i, j)=L(1) \cdot \hat{X}(1)+L(2) \cdot \hat{X}(2)+ \\
9.1 & L(3) . \hat{X}(3)+L(4) \cdot \hat{X}(4)
\end{array}
$$

9.2 Return to step 1 for the next $(i, j)$ 10 - Increase the window by $5 \times 5$.

11- Eliminate the noisy pixels within the window.

12- Insert the noise free pixels into vector $S$.

13- Assign the number of pixels in the $S$ to $P$.

14- If $(P<1)$, go to step 19

15- If $(P=1)$

15.1 Let $g(i, j)$ equal to the pixel value in $P$

15.2 Return to step 1 for the next $(i, j)$

16- I $(P=2)$

$16.1 g(i, j)=P(1) \cdot X(1)+P(2) \cdot X(2)$

16.2 Return to step 1 for the next $(i, j)$

17- If $(P=3)$

$17.1 g(i, j)=P(1) \cdot \bar{X}(1)+P(2) \cdot \bar{X}(2)+P(3) \cdot \bar{X}(3)$

17.2 Return to step 1 for the next $(i, j)$

18- If $(P \geq 4)$

$$
\begin{aligned}
& \quad g(i, j)=P(1) . \hat{X}(1)+P(2) \cdot \hat{X}(2)+ \\
& 18.1 P(3) . \hat{X}(3)+P(4) . \hat{X}(4) \\
& 18.2 \text { Return to step } 1 \text { for the next }(i, j)
\end{aligned}
$$

19- Replace $g(i, j)$ with the nearest noise free pixel.

20 -Return to step 1 for the next $(i, j)$

\section{SIMULATION RESULTS}

To check the efficiency of the proposed technique, a variety of well-known test images with 1024X1024: Bridge, Boat, Goldhill, and Peppers are used. A wide range of high noise ratios varied from $50 \%$ to $97 \%$ is tested. Haidi at el method [14], DBA [18], MDBUTMF [19] and standard median filter (SMF) are used in the comparison with the proposed algorithm (PA). Denoising performance is quantitatively measured by the peak signal-to-noise ratio (PSNR) as defined in (1)

$P S N R=10 \log _{10}\left(\frac{(255)^{2}}{M S E}\right)$

$M S E=\frac{\sum_{j} \sum_{j}(F(i, j)-\hat{F}(i, j))^{2}}{M \times N}$ where MSE stands for mean square error, $M \times N$ is size of the image, $F$ represents the original image and $\hat{F}$ denotes the denoised image. Table I shows PSNR value of the proposed algorithm (PA) against the existing algorithms by varying the noise density from $50 \%$ to $97 \%$. Thus, it is observed that the proposed method gives better PSNR than other existing algorithm. Figure.2 shows a plot of PSNR versus noise densities for Bridge, Boat, Goldhill, and Peppers images. The visual result of the proposed method for Boat image corrupted by 50\%, Goldhill image corrupted by $80 \%$ and Peppers image corrupted by 97\% are shown in Figure.3, Figure.4 and Figure.5, respectively.

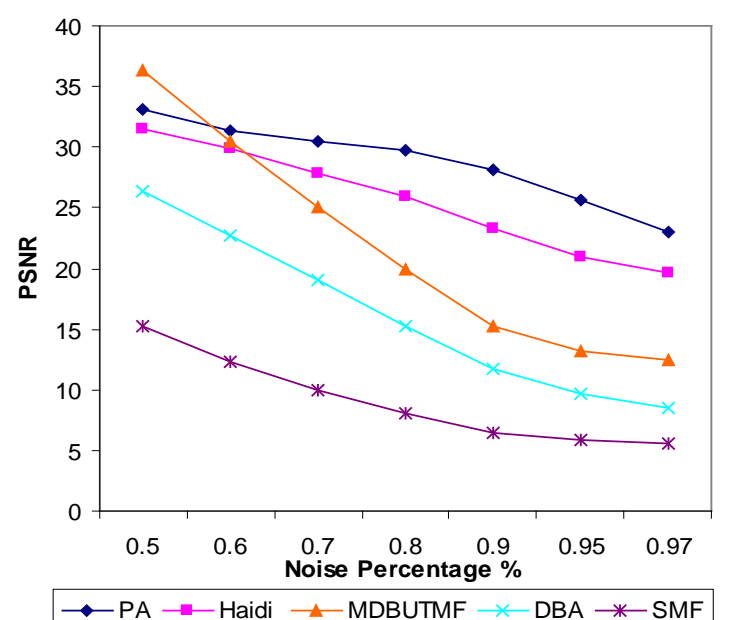

(a) Comparison graph of PSNR at different noise densities for Bridge image.

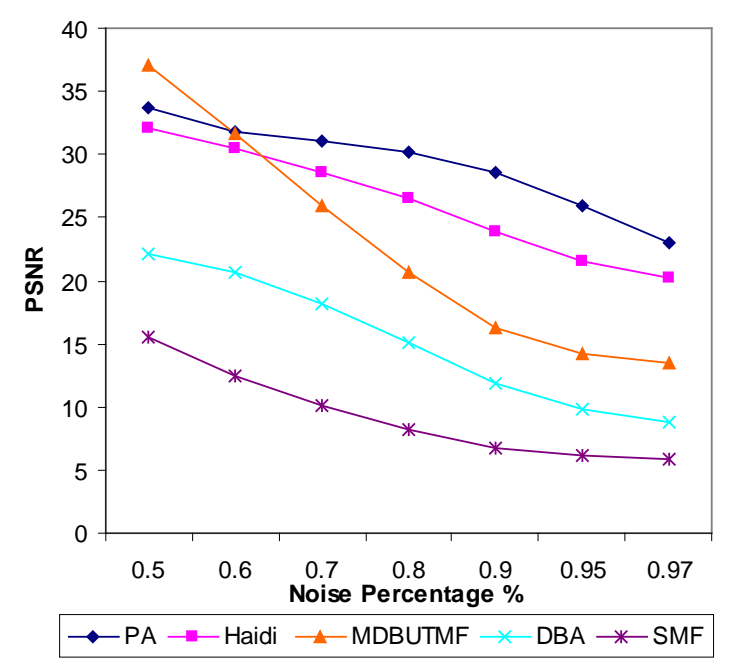

(b) Comparison graph of PSNR at different noise densities for Boat image. 


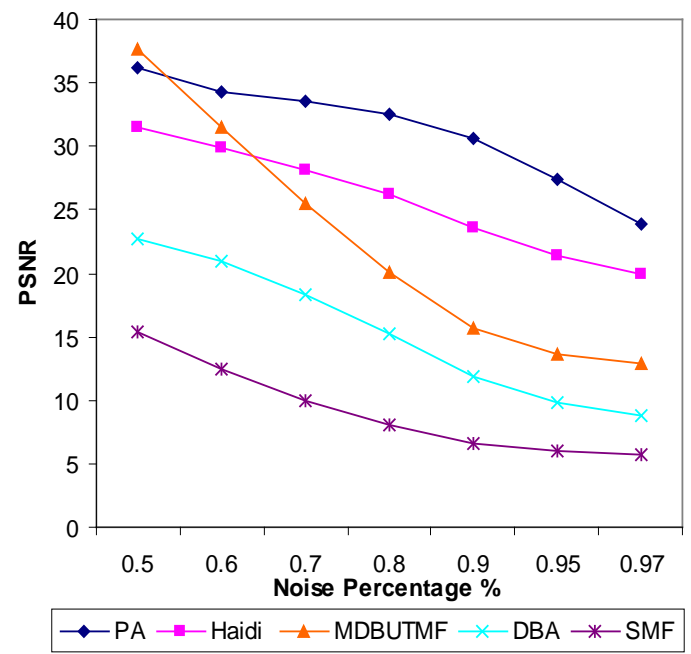

(c) Comparison graph of PSNR at different noise densities for Goldhill image.

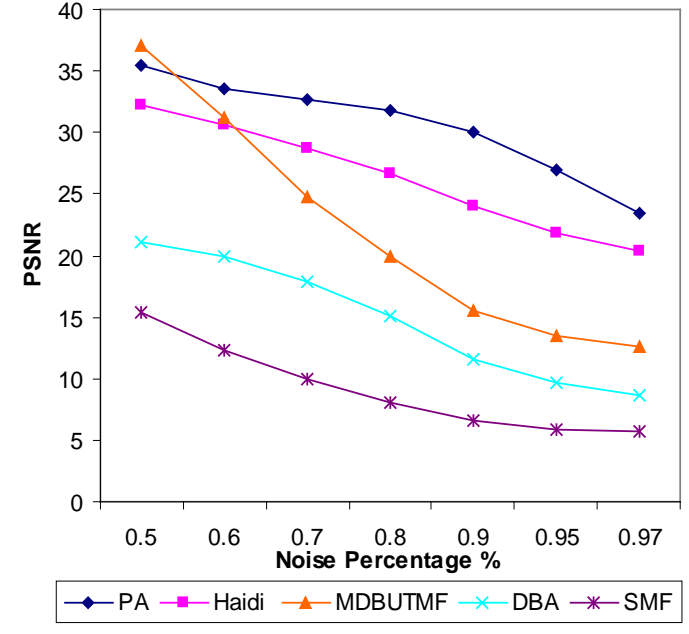

(d) Comparison graph of PSNR at different noise densities for Peppers image.

Figure.2 Comparison Graph of PSNR at Different Noise Densities for Bridge, Boat, Goldhill and Peppers Images

Table I : COMPARISON OF PROSSING RESULTS IN PSNR (dB) FOR BRIDGE, BOAT, GOLDHILL AND PEPPERS IM AGES.

\begin{tabular}{|c|c|c|c|c|c|c|c|}
\hline \multicolumn{8}{|c|}{ Bridge } \\
\hline & $50 \%$ & $60 \%$ & $70 \%$ & $80 \%$ & $90 \%$ & $95 \%$ & $97 \%$ \\
\hline SMF & 15.2561 & 12.2568 & 9.9091 & 7.9927 & 6.4850 & 5.8551 & 5.6152 \\
\hline DBA [18 ] & 26.3414 & 22.6679 & 19.0508 & 15.2777 & 11.7214 & 9.6453 & 8.5225 \\
\hline Haidi [14] & 31.4620 & 29.9041 & 27.7907 & 25.9067 & 23.3135 & 21.0217 & 19.6282 \\
\hline MDBUTMF[19] & 36.3076 & 30.4300 & 24.9938 & 19.8859 & 15.3091 & 13.2396 & 12.4492 \\
\hline $\mathrm{PA}$ & 33.0957 & 31.2863 & 30.4471 & 29.6731 & 28.1027 & 25.6687 & 22.9920 \\
\hline \multicolumn{8}{|c|}{ Boat } \\
\hline SMF & 15.4695 & 12.5225 & 10.1506 & 8.2364 & 6.7249 & 6.0882 & 5.8294 \\
\hline$\overline{\text { DBA [18 ] }}$ & 22.1797 & 20.7020 & 18.2161 & 15.1558 & 11.8583 & 9.8329 & 8.7985 \\
\hline Haidi [14] & 32.0482 & 30.4337 & 28.5767 & 26.5532 & 23.8427 & 21.5303 & 20.2233 \\
\hline MDBUTMF[19] & 37.1259 & 31.7201 & 25.9988 & 20.5980 & 16.2560 & 14.2292 & 13.4333 \\
\hline $\mathrm{PA}$ & 33.6924 & 31.8605 & 31.0683 & 30.1884 & 28.5687 & 25.9500 & 23.0467 \\
\hline \multicolumn{8}{|c|}{ Goldhill } \\
\hline SMF & 15.3552 & 12.3908 & 10.0116 & 8.0830 & 6.5889 & 5.9580 & 5.7137 \\
\hline DBA [18 ] & 22.7533 & 20.9759 & 18.3288 & 15.2281 & 11.8048 & 9.7816 & 8.7662 \\
\hline Haidi [14] & 31.4529 & 29.8889 & 28.1578 & 26.1550 & 23.5644 & 21.3336 & 19.9978 \\
\hline MDBUTMF[19] & 37.6932 & 31.4600 & 25.5095 & 20.0399 & 15.7391 & 13.6901 & 12.8968 \\
\hline $\mathrm{PA}$ & 36.2110 & 34.3507 & 33.5131 & 32.5638 & 30.6643 & 27.4035 & 23.9013 \\
\hline \multicolumn{8}{|c|}{ Peppers } \\
\hline SMF & 15.3275 & 12.3661 & 9.9594 & 8.0579 & 6.5276 & 5.8868 & 5.6543 \\
\hline DBA [18 ] & 21.1612 & 19.9230 & 17.8101 & 15.0331 & 11.6172 & 9.6086 & 8.6595 \\
\hline Haidi [14] & 32.2007 & 30.6334 & 28.6588 & 26.6614 & 24.0642 & 21.8485 & 20.3038 \\
\hline MDBUTMF[19] & 37.0994 & 31.1429 & 24.7732 & 19.9782 & 15.5061 & 13.4513 & 12.6471 \\
\hline $\mathrm{PA}$ & 35.3872 & 33.4932 & 32.6362 & 31.7422 & 30.0189 & 26.9156 & 23.4969 \\
\hline
\end{tabular}




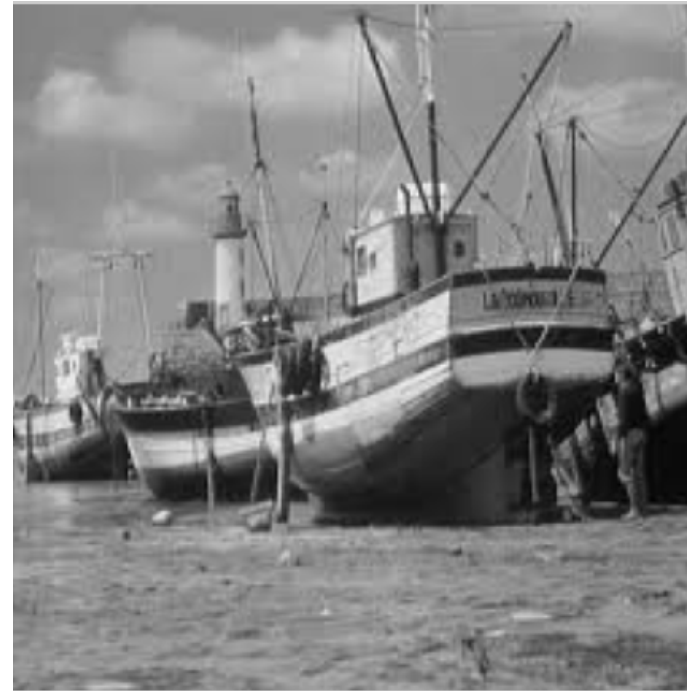

a) Original Boat image

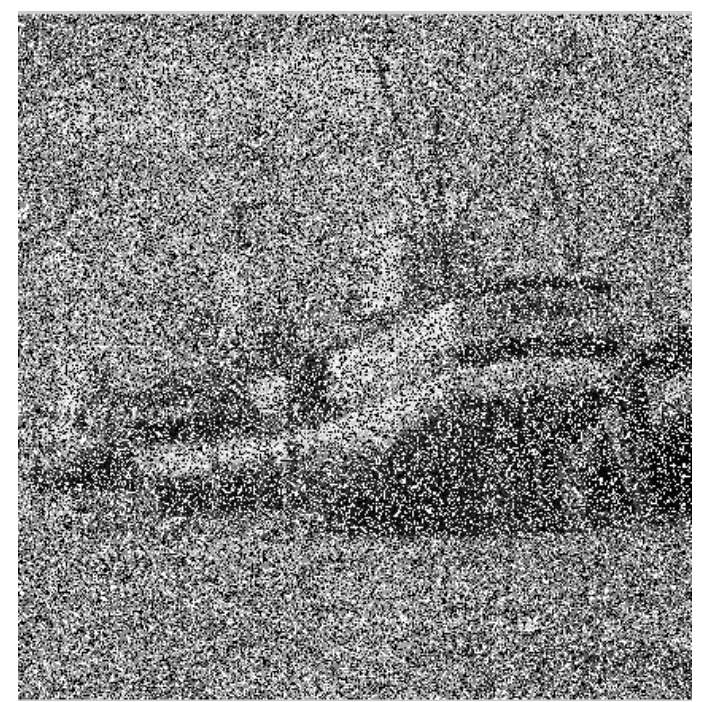

(b) Boat image corrupted by $50 \%$

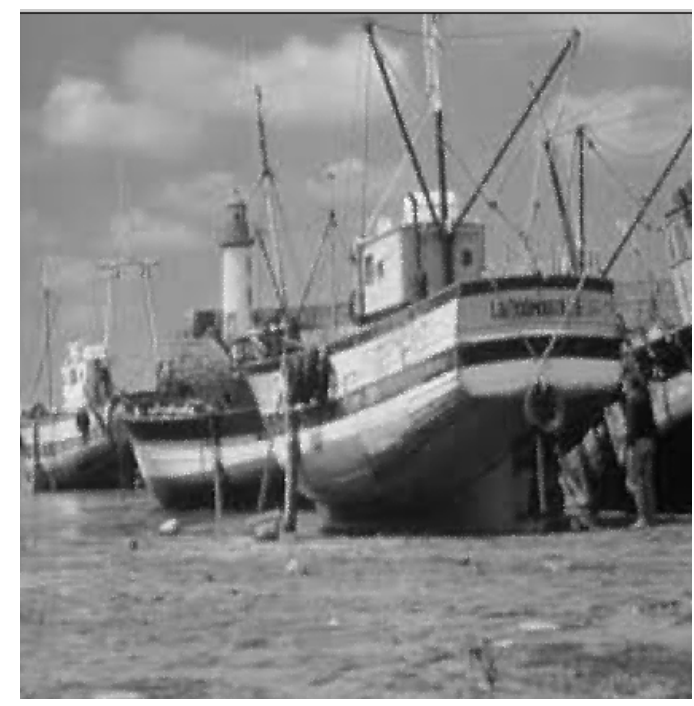

(c) The result of PA for corrupted image in (b) Figure 3. The Result of PA for Corrupted Boat Image

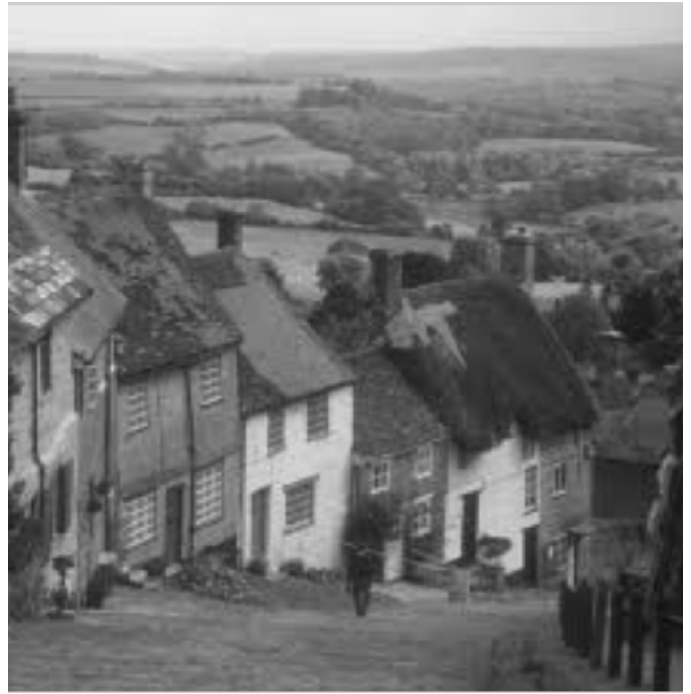

(a) Original Goldhill image

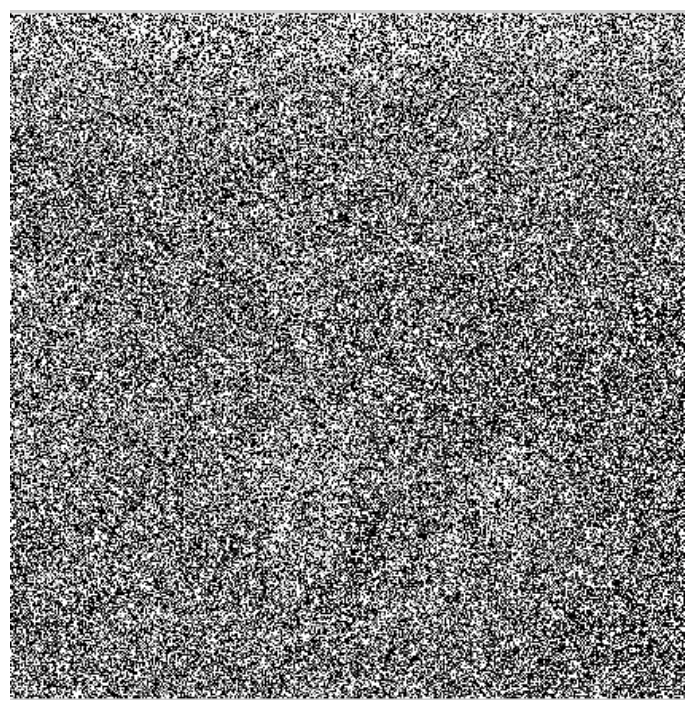

(b) Goldhill image corrupted by $80 \%$

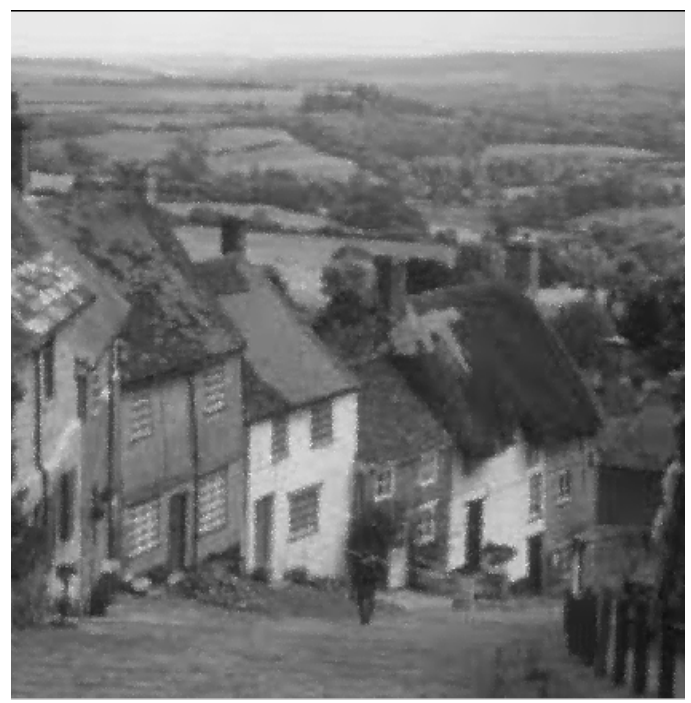

(c) The result of PA for corrupted image in (b)

Figure 4. The Result of PA for Corrupted Goldhill Image 


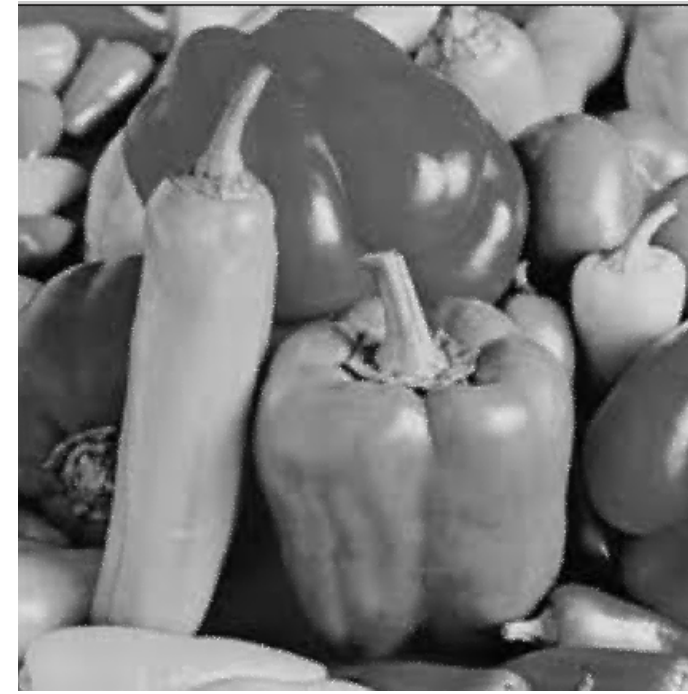

(a) Original Peppers image

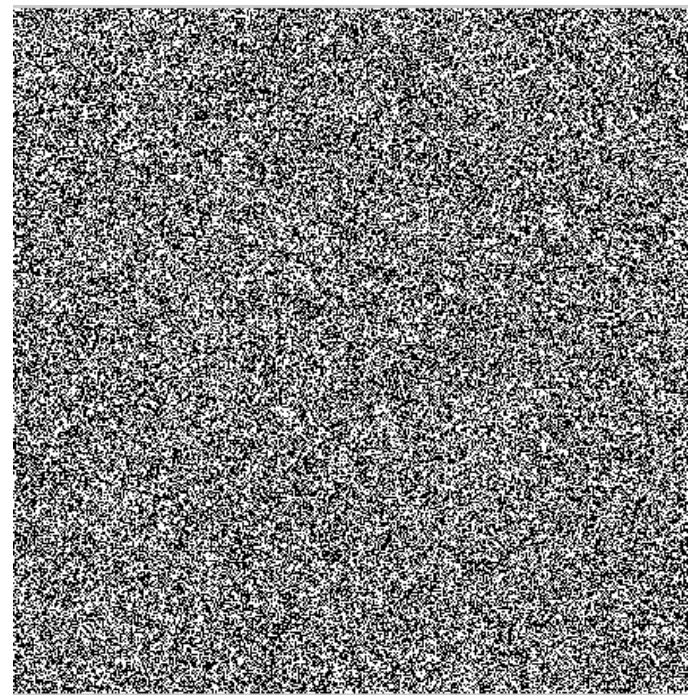

(b) Peppers image corrupted by $97 \%$

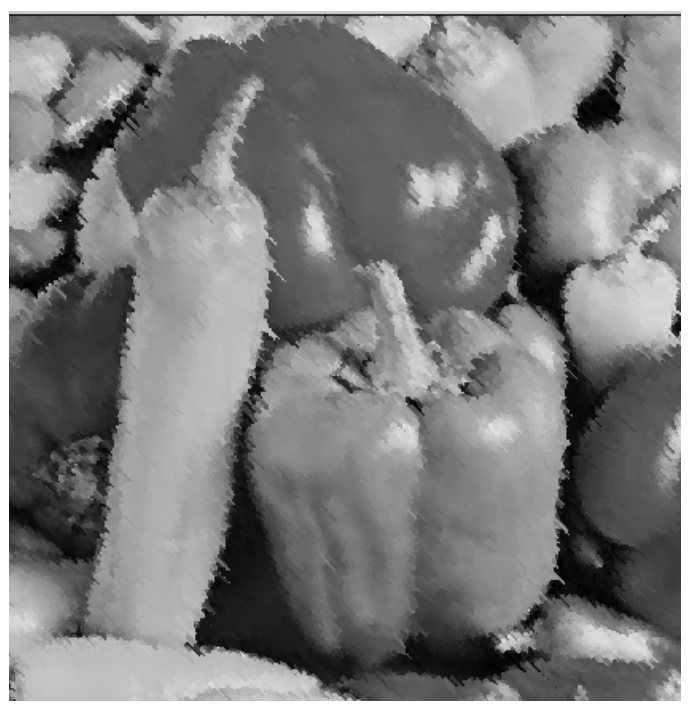

(c) The result of PA for corrupted image in (b) Figure 5. The Result of PA for Corrupted Peppers Image

\section{CONCLUSIONS AND PROSPECTS}

A new denoising method for removing salt-andpepper noise is introduced in this paper. This method is simple in implementation where no complex operations are required. The proposed method framework outperforms other existing denoising method for removal of salt - and - pepper noise with preserving image details as it is clearly seen in visual and quantitative results. Moreover, the suggested method presents a quite stable performance over a wide variety of images. In addition, the presented method does not need large data set for training like artificial neural networks and genetic programming, which consuming time for training. Indeed one image is enough for extracting the coefficients, which uses in the filter action. The main advantage of this method is no adjustment of threshold parameters, which influences the behavior of the system.

\section{REFERENCES}

[1] T.Veerakumar, S.Esakkirajan and Ila Vennila, "Salt and Pepper noise removal in video using adaptive decision based median filter," International Conference on Multimedia, Signal Processing and Communication Technologies ,pp. 87:90, 2011.

[2] S. Huang and J. Zhu, "Removal of salt-and-pepper noise based on compressed sensing," Electronics Letters Vol. 46, No. 17, 19th August 2010.

[3] Kenny Kal Vin Toh, Haidi Ibrahim and Muhammad Nasiruddin Mahyuddin, "Salt-andPepper noise detection and reduction using fuzzy switching median filter," IEEE Trans. Consumer Electron., Vol. 54, No. 4, pp. 1956:1961, Nov. 2008.

[4] Pei-Yin Chen and Chih-Yuan Lien, “An efficient edge-preserving algorithm for removal of saltand-pepper noise,” IEEE Signal Process. Lett., Vol. 15, pp. 833-836, 2008.

[5] Cangju Xing, "An effective method for removing heavy Salt-and-Pepper noise,” Congress on Image and Signal Processing, 2008.

[6] Yi Hong, Sam Kwong and Hanli Wang, "Decisionbased median filter using K-nearest noise-free pixels,” pp.1193:1196, ICASSP 2009.

[7] Umesh Ghanekar, “A contrast enhancement-based filter for removal of random valued impulse noise,” IEEE Signal Process. Lett., Vol. 17, No. 1, pp. 47-50, Jan. 2010.

[8] J. Astola and P. Kuosmanen, "Fundamentals of Nonlinear Digital Filtering,” Boca Raton, FL: CRC, 1997.

[9] S.-J. Ko and Y.-H. Lee, “Center weighted median filters and their applications to image enhancement," IEEE Trans. Circuits Syst., vol. 38, pp. 984-993, Sep. 1991.

[10] G. Arce, J. Paredes, "Recursive weighted median filters admitting negative weights and their 
Optimization, " IEEE Trans. Signal Proc., Vol. 48, pp 768-779, 2000.

[11] Kenny Kal Vin Toh and Nor Ashidi Mat Isa, "Noise adaptive fuzzy switching median filter for Salt-and-Pepper noise reduction,” IEEE Signal Process. Lett., Vol. 17, No. 3, pp. 281-284, Mar. 2010.

[12] X.M. Zhang, Z.P. Yin and Y.L. Xiong, “Adaptive switching mean filter using conditional morphological noise detector," Electron. Lette, Vol. 44, No. 6, 13th Mar. 2008.

[13] WANG Chang-you, LI Lin-lin, YANG Fu-ping, and GONG Hui,, "A new kind of adaptive weighted median filter algorithm," International Conference on Computer Application and System Modeling (ICCASM 2010).

[14] Haidi Ibrahim, Nicholas Sia Pik Kong, and Theam Foo Ng "Simple adaptive median filter for the removal of impulse noise from highly corrupted images," IEEE Trans. Consumer Electron., Vol. 54, No. 4, pp. 1920-1927, Nov. 2008.

[15] Wenbin Luo, "Efficient removal of impulse noise from digital images," IEEE Trans. Consumer Electron., Vol. 52, No. 2, pp. 523- 527, May 2006.

[16] Shuqun Zhang and Mohammad A. Karim, "A new impulse detector for switching median filters," IEEE Signal Process. Lett., Vol. 9, No. 11, pp. 360-363, Nov. 2002.

[17] H. Hwang, and R. A. Haddad, "Adaptive median filters: New algorithms and results,” IEEE Trans. Image Process., Vol. 4, No. 4, pp. 499-502, Apr. 1995.

[18] K. S. Srinivasan and D. Ebenezer, “A new fast and efficient decision-based algorithm for removal of high-density impulse noises," IEEE Signal Process. Let, Vol. 14, No. 3, pp. 189-192, Mar. 2007.

[19] S. Esakkirajan, T. Veerakumar, Adabala N. Subramanyam, and C. H. PremChand, "Removal of high density salt and pepper noise through modified decision based unsymmetric trimmed median filter," IEEE Signal Process. Lett., Vol. 18, No. 5, pp. 287-290, May. 2011.

Hani M. Ibrahem was born in Egypt on Septamper 12th1978. He received the M.S. and Ph.D degrees in Computer Science at the University of Menoufyia, Egypt in 2004 and 2008, respectively. His research interests lies in the areas of image processing. Currently he is a lecture of Computer Science in the Faculty of Science, at the University of Menoufyia, Egypt. 